\title{
OFFLINE SigNATURE VERIFICATION USING SUPPORT LOCAL BINARY PATTERN
}

\author{
P.Vickram, Dr. A. Sri Krishna and D.Swapna \\ Department of Computer Science \& Engineering, R.V. R \& J.C College of Engineering, \\ Guntur
}

\begin{abstract}
The offline signature verification is an automatic verification system that works on the scanned image of a signature. Signature verification uses the gray level measure with varying foreground features. The signature verification is performed by identifying feature vector using local patterns. The Local Binary Pattern (LBP) in signature verification has used to extract the local structure information by establishing the relationship between central pixel and adjacent pixels. This paper uses the Support Local Binary Pattern (SLBP) features for signature verification. The signatures are tested on MCYT dataset. The accuracy of the proposed method is tested against k-Nearest Neighbor Classifier (KNNC) and Linear Discriminant Classifier (LDC).
\end{abstract}

\section{KEYWORDS}

Histogram, Completed Local Binary Pattern (CLBP), Support Local Binary Pattern (SLBP).

\section{INTRODUCTION}

A biometric system is used for authentication systems and personal verification [1]. One of the most important traits in biometric system is handwritten signatures [2].Signature verification is a uniquely identifying system. It can be categorized in two different ways. Off-line signature verification deals with shape only. This mode is also known as static. Online signature verification deals with changing features of like speed, pen pressure, directions, stroke length, and when the pen is lifted from the paper. This mode is also known as dynamic. Signature verification is to evaluate the dependence of the gray level based features [3] and strategies are proposed to improve their robustness to gray level distortion and segmentation errors. Like Local Binary Patterns (LBP) [4], Local Directional Patterns (LDP) [5] is a micro pattern representation which is modelled by histogram to preserve the information about the distribution of the LDP micro patterns. Texture measures are proposed for offline automatic signature verification based on histograms of completed local binary and support local binary patterns. By using k-nearest neighbor (KNNC) and Linear Discriminant Classifier (LDC) these feature vectors are evaluated. This paper uses the SLBP for signature verification.

This paper is organized as follows. Section 2 discusses the completed local binary pattern in detail. Section 3 discusses the support local binary pattern. Section 4 discusses Experiment and simulation evaluation. Results and discussions are given in section 5 and finally conclusion is in section 6 . 


\section{Completed local Binary Pattern}

Completed Local Binary Pattern (CLBP) [6], has three components, sign, magnitude and center pixel. CLBP-S represents the sign either positive or negative which indicates the difference between the centre pixel and local pixel, CLBP-M represents the magnitude which indicates the difference between the centre pixel and local pixel and CLBP-C represents the center value which indicates the difference between local pixel value and average central pixel value. CLBP-S is normal LBP. CLBP successfully captures more discriminative information by combining the sign, magnitude, and center gray level, as shown in figure. 1.

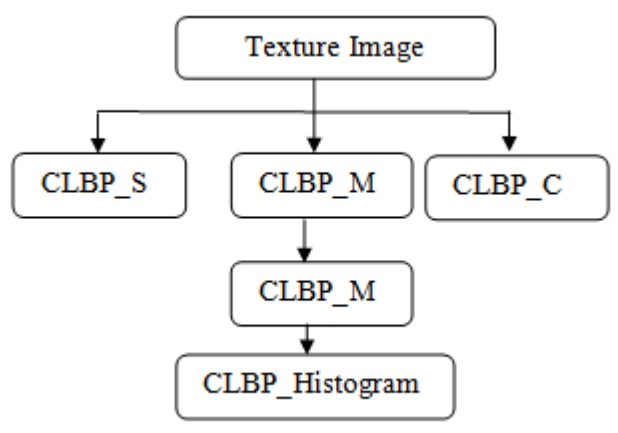

Fig 1: Frame Work of CLBP

The operator $\mathrm{S}$ is estimated as:

$$
\begin{aligned}
\text { CLBP_S } \mathrm{S}_{\mathrm{P}, \mathrm{R}} & =\sum_{p=0}^{p-1} 2^{p} t(\mathrm{ip}-\mathrm{ic}) \\
\mathrm{t}_{\mathrm{p}} & =\left\{\begin{array}{l}
1, i p \geq i c \\
0, t p<i c
\end{array}\right.
\end{aligned}
$$

where ic and ip are the gray values of the central pixel and pth neighbor pixel,respectively.

The operator $\mathrm{M}$ is estimated as:

$$
\begin{gathered}
\mathrm{CLBP}_{-} \mathrm{M}_{\mathrm{P}, \mathrm{R}}=\sum_{p=0}^{p-1} 2^{p} \mathrm{t}(\mathrm{mp}, \mathrm{c}) \\
\mathrm{t}(\mathrm{mp}, \mathrm{c})=\left\{\begin{array}{l}
1,|i p-i c| \geq c \\
0,|i p-i c|<c
\end{array}\right.
\end{gathered}
$$

where $\mathrm{mp}$ is the difference between central pixel and neighbor pixel and $\mathrm{c}$ is the average value of an entire image

The operator $\mathrm{C}$ is coded as

$$
\text { CLBP_C } \mathrm{P}_{\mathrm{R}, \mathrm{R}}=\mathrm{t}\left(\mathrm{z}_{\mathrm{c}}, \zeta\right)
$$

where, $\zeta$ mean gray level of the entire image and $z_{c}$ is the central pixel.

where, $\mathrm{R}$ is the radius of the neighborhood and $\mathrm{P}$ is the neighborhood pixels. 


\section{Support Local Binary Pattern}

In support local binary pattern (SLBP) [7], it extracts the information by establishing the relationship among the neighboring pixels to improve the performance of current Local Patterns. The SCLBP is then generated by joining the histogram of CLBP in nine directions. The joining of the histograms in two directions: $\left[45^{\circ}, 90^{\circ}\right],\left[135^{\circ}, 180^{\circ}\right],\left[225^{\circ}, 270^{\circ}\right]$, and $\left[315^{\circ}, 360^{\circ}\right]$, gives the results, CLBP [1, 2], CLBP [3, 4], CLBP [5, 6], and CLBP [7, 8] respectively. By joining the histograms of CLBP [1,2] and CLBP [3,4] gives the result, CLBP [1, 4]. By joining the histograms of CLBP [5,6] and CLBP [7, 8] gives the result, CLBP [5, 8]. By joining the histograms of CLBP [1, 4] and CLBP [5, 8] gives the result, CLBP [1, 8].Finally, SCLBP is obtained by joining the histograms of CLBP at direction $0^{\circ}$ and CLBP $[1,8]$, as shown in figure 2 .

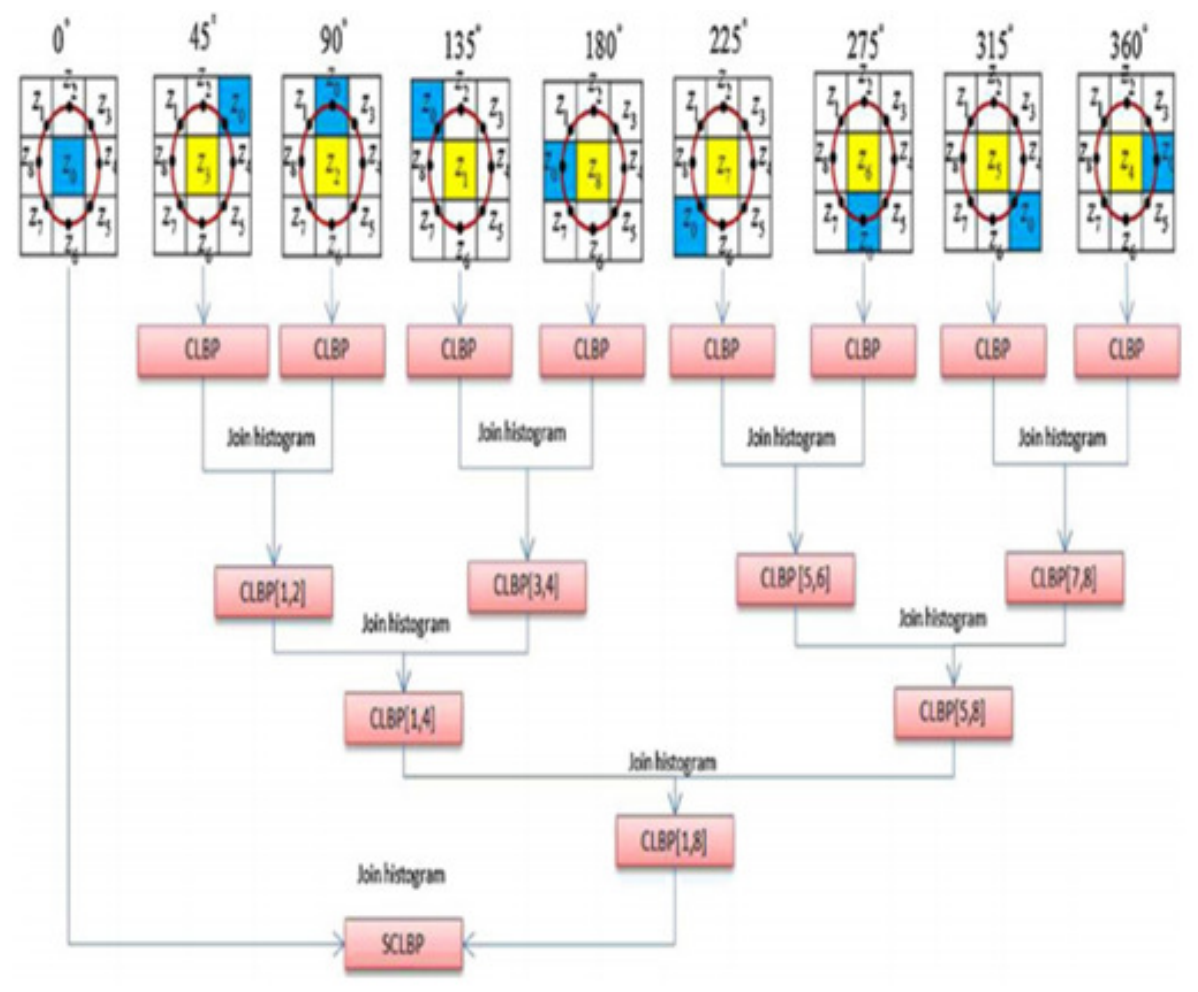

Fig 2: Proposed Model of SLBP with CLBP for Texture Classification (SCLBP)

\section{EXPERIMENT AND Simulation Evaluation}

The MCYT database is used for the signature verification [8]. From four different Spanish sites it includes totally 75 signers. For each signer the database includes 15 genuine signatures and 15 simulated forgeries. In two sessions, genuine signatures were acquired. To imitate the shape forgers are given the signature images of clients after training with them several times. All the signatures in the signature database were acquired with the same inking pen. 12 bank checks and 8 invoices with different background complexity, totally 20 images are included in check database. Some of the checks with signatures are shown in figure 3 and figure 4 respectively. 
International Journal of Artificial Intelligence and Applications (IJAIA), Vol. 7, No. 6, November 2016
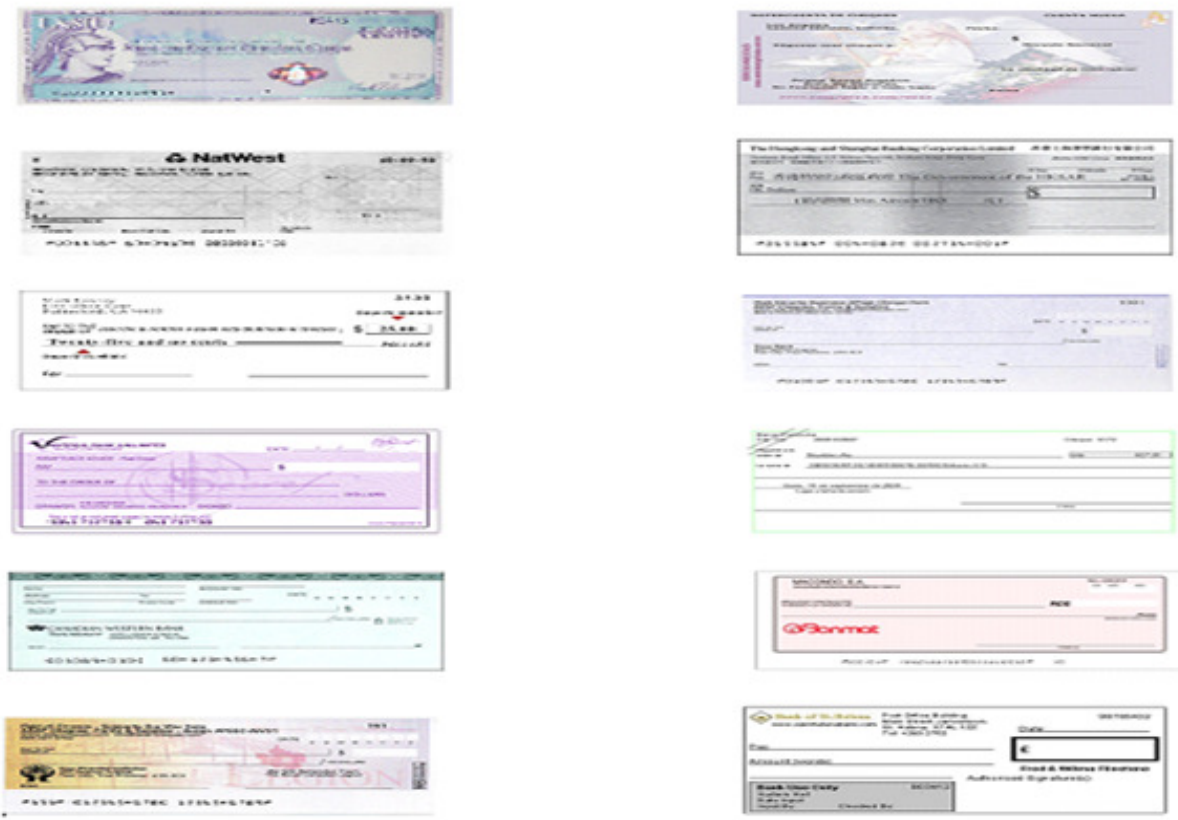

Fig 3: Different Checks in Check Database

\section{Training SeT}
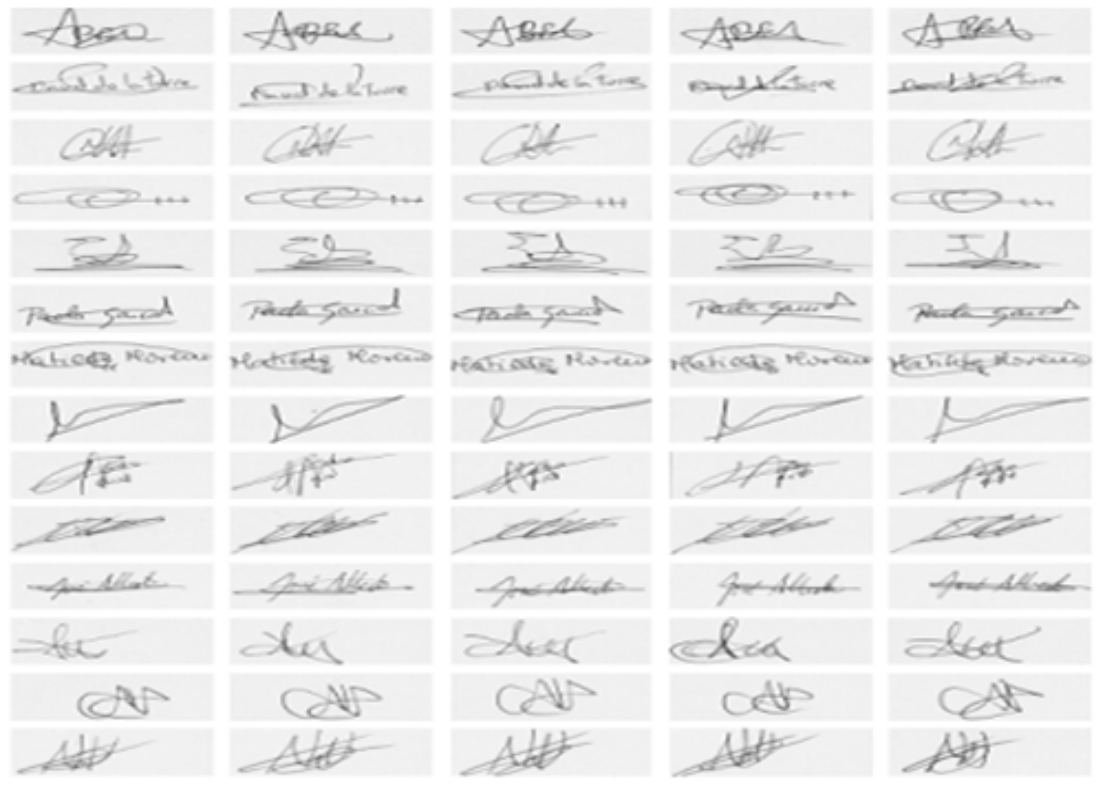

Fig 4: Different Signatures in MCYT Dataset

Signature verification of scanned image with complex background includes several steps, which usually begin with preprocessing, feature extraction using various local patterns and 
International Journal of Artificial Intelligence and Applications (IJAIA), Vol. 7, No. 6, November 2016

classification. A MCYT database has been used in all the experiments. The Flow chart of signature verification is shown below.

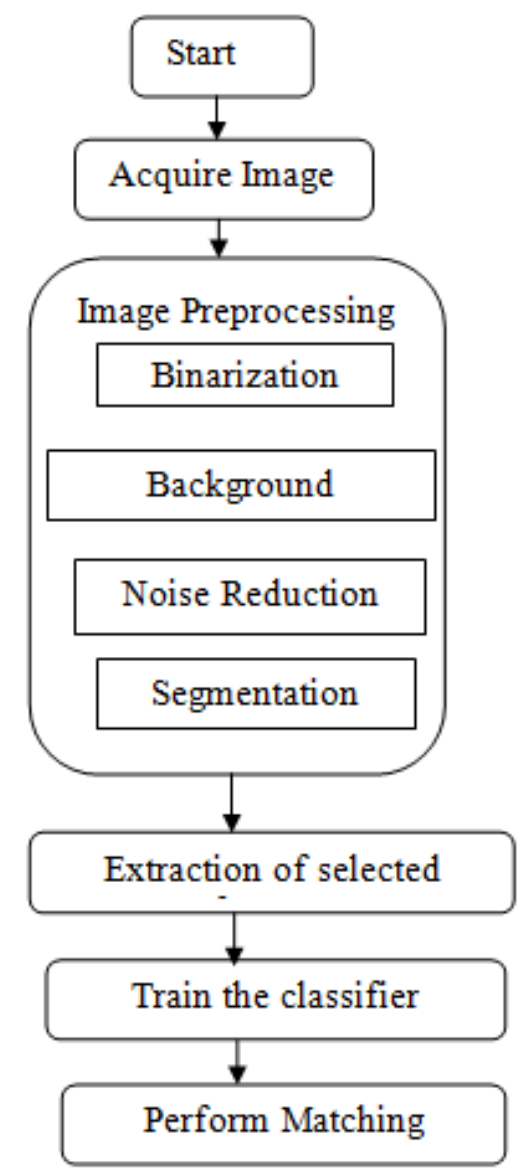

Fig 5: Flow Chart of Signature Verification.

The process adopted for preprocessing is given in algorithm 1 and the process for classification is given in algorithm 2.

\section{Algorithm 1: Preprocessing}

Input: Signature image with Complex background.

Output: Preprocessed image (Back ground and Noise Removal).

Methodology:

Step 1: Read signature image and convert it into gray scale image.

Step 2: The image which is from the step 1 is converted in to binary image using posterization [3]

Step 3: Remove the noise (strokes) from the binarized image.

Step 4: Segmentation of Original Image by using noise Removal Image as mask. 


\section{Algorithm 2: Classification}

Step1: In feature extraction, the features of the signature image are extracted using histogram of local patterns.

Step 2: In Classification, the signature features are extracted with different classifiers, k-nearest neighbor (KNNC) and Linear Discriminant Classifier (LDC).

Step 3: If the signature features are matched with the database then it is classified as genuine otherwise forge.

A confusion matrix [9] is used to evaluate the performance of an algorithm. Rows correspond to classes which are true labels. Columns correspond to classes which are estimated labels. The diagonal elements in the matrix represent the number of correctly classified pixels of each class.

\section{RESUlts AND DiscuSSIONS}

\subsection{CLBP}

The CLBP is analyzed on the sample signature image and the CLBP sign, magnitude and center gray level value images are shown in figure 6 . The histogram feature vectors are calculated using CLBP histogram are shown in figure 7.These histogram feature vectors are evaluated with different classifiers, k-nearest neighbor (KNNC )and Linear Discriminant Classifier(LDC). In experiment, 100 sample signatures are taken from MCYT database and 15 individual signatures are from each sample total 1500 signatures are taken as training set (some of the examples of signature samples in training set are shown above). The testing of CLBP (feature extraction) is carried out using training set. Results of classifiers are shown in table I and table II.

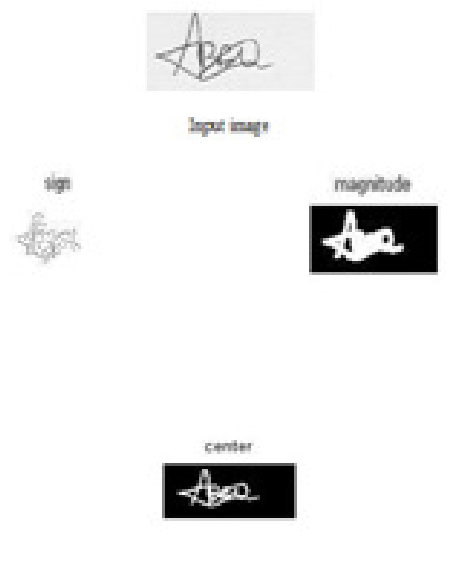

Fig 6: Images of CLBP

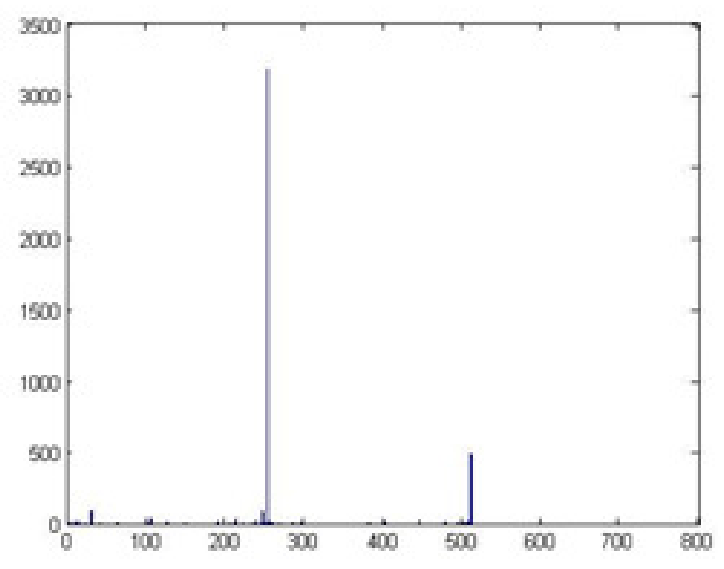

Fig 7: CLBP Histogram 
International Journal of Artificial Intelligence and Applications (IJAIA), Vol. 7, No. 6, November 2016

Table I Confusion matrix with Linear Discriminant Classifier (LDC)

\begin{tabular}{|l|l|l|l|l|l|l|l|}
\hline $\begin{array}{l}\text { True } \\
\text { labels }\end{array}$ & 1 & 2 & 3 & 4 & 5 & 6 & Totals \\
\hline 1 & 12 & 3 & 0 & 0 & 0 & 0 & 15 \\
\hline 2 & 1 & 10 & 0 & 0 & 0 & 4 & 15 \\
\hline 3 & 0 & 0 & 15 & 0 & 0 & 0 & 15 \\
\hline 4 & 0 & 0 & 0 & 13 & 0 & 2 & 15 \\
\hline 5 & 0 & 0 & 0 & 2 & 13 & 0 & 15 \\
\hline 6 & 1 & 4 & 0 & 2 & 0 & 8 & 15 \\
\hline Totals & 14 & 17 & 15 & 17 & 13 & 14 & 90 \\
\hline
\end{tabular}

Table II Confusion Matrix with K-Nearest Neighbour Classifier (KNNC)

\begin{tabular}{|l|l|l|l|l|l|l|l|}
\hline $\begin{array}{l}\text { True } \\
\text { labels }\end{array}$ & 1 & 2 & 3 & 4 & 5 & 6 & Totals \\
\hline 1 & 14 & 1 & 0 & 0 & 0 & 0 & 15 \\
\hline 2 & 2 & 12 & 0 & 0 & 0 & 1 & 15 \\
\hline 3 & 0 & 0 & 15 & 0 & 0 & 0 & 15 \\
\hline 4 & 0 & 0 & 0 & 15 & 0 & 0 & 15 \\
\hline 5 & 0 & 0 & 0 & 0 & 2 & 13 & 15 \\
\hline 6 & 3 & 1 & 0 & 1 & 0 & 10 & 15 \\
\hline Totals & 19 & 14 & 15 & 18 & 13 & 11 & 90 \\
\hline
\end{tabular}

\subsection{SLBP}

The SLBP is analyzed on the sample signature images. The histogram feature vectors are calculated using SLBP histogram as shown in figure 8.These histogram feature vectors are evaluated with different classifiers, k-nearest neighbor (KNNC) and Linear Discriminant Classifier (LDC). In experiment, 100 sample signatures are taken from MCYT database and 15 individual signatures are from each sample total 1500 signatures are taken as training set (some of the examples of signature samples in training set are shown above). The testing of SLBP (feature extraction) is carried out using training set. Results are of classifiers are shown in table III and table IV.

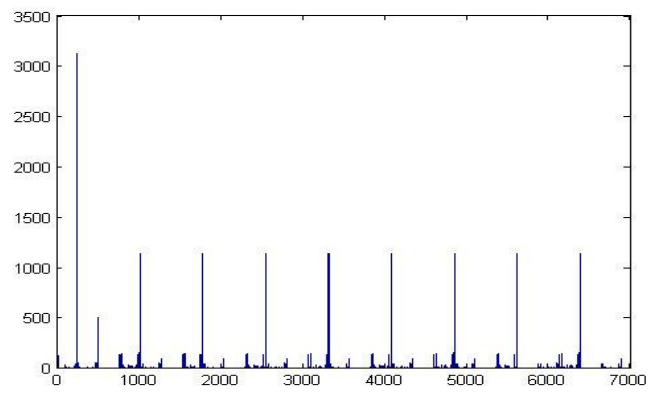

Fig 8 SLBP Histogram 
International Journal of Artificial Intelligence and Applications (IJAIA), Vol. 7, No. 6, November 2016

Table III Confusion Matrix with Linear Discriminant Classifier (LDC)

\begin{tabular}{|l|l|l|l|l|l|l|l|}
\hline $\begin{array}{l}\text { Tru } \\
\text { What }\end{array}$ & 1 & 2 & 3 & 4 & 5 & 5 & Thes \\
\hline 1 & 12 & 5 & 0 & 0 & 0 & 0 & 15 \\
\hline 2 & 1 & 10 & 0 & 0 & 0 & 4 & 15 \\
\hline 3 & 0 & 0 & 15 & 0 & 0 & 0 & 15 \\
\hline 4 & 0 & 0 & 0 & 15 & 0 & 15 & 15 \\
\hline 5 & 0 & 0 & 0 & 2 & 13 & 0 & 15 \\
\hline 6 & 1 & 1 & 0 & 0 & 0 & 13 & 15 \\
\hline Toull & 14 & 14 & 15 & 15 & 13 & 19 & 90 \\
\hline
\end{tabular}

Table IV Confusion Matrix with K-Nearest Neighbour Classifier (KNNC)

\begin{tabular}{|l|l|l|l|l|l|l|l|}
\hline $\begin{array}{l}\text { True } \\
\text { labels }\end{array}$ & 1 & 2 & 3 & 4 & 5 & 6 & Totals \\
\hline 1 & 14 & 1 & 0 & 0 & 0 & 0 & 15 \\
\hline 2 & 2 & 12 & 0 & 0 & 0 & 1 & 15 \\
\hline 3 & 0 & 0 & 15 & 0 & 0 & 0 & 15 \\
\hline 4 & 0 & 0 & 0 & 15 & 0 & 0 & 15 \\
\hline 5 & 0 & 0 & 0 & 0 & 2 & 13 & 15 \\
\hline 6 & 3 & 1 & 0 & 1 & 0 & 10 & 15 \\
\hline Totals & 19 & 14 & 15 & 18 & 13 & 11 & 90 \\
\hline
\end{tabular}

SLBP has less error rate as compared to the Completed Local Binary Pattern (CLBP) using LDC classifier and it has same error rate as compared to the Completed Local Binary Pattern (CLBP) using KNN classifier. Comparisons of CLBP and SLBP with their error rate are shown in table V.

Table V Error Rate of Local Patterns using KNNC Classifier and LDC Classifier

\begin{tabular}{|l|l|l|}
\hline Loca1 patterns & LDC & KNNC \\
\hline CLBP & 0.3000 & 0.4000 \\
\hline SLBP & & \\
\hline
\end{tabular}


International Journal of Artificial Intelligence and Applications (IJAIA), Vol. 7, No. 6, November 2016

\section{CONCLUSION}

The algorithm is tested on MCYT database. The offline automatic signature verification is performed using SLBP features. In the experiment same features have been used for both CLBP and SLBP for signature verification. These feature vectors were evaluated using k- nearest neighbour and Linear Discriminant Classifiers. It has been observed that the CLBP and SLBP gives the same results for KNN classifier but SLBP gives less error rate as compared to the CLBP if we are using Linear Discriminant Classifier.

\section{REFERENCES}

[1] D. Impedovo and G. Pirlo, "Automatic signature verification: The stateof the art," IEEE Trans. Syst.ManCybern.-Part C: Applications Rev., vol. 38, no. 5, pp. 609-635, Sep. 2008.

[2] W. Hou, X. Ye, and K. Wang, "A survey of off-line signature verification," in Proc. Int. Conf. Intelligent Mechatronics Automation, Chengdu, China, Aug. 2004, pp. 536-541.

[3] J. F. Vargas, M. A. Ferrer, C. M. Travieso, and J. B. Alonso, "Offline signature verification based on grey level information using texture features," Pattern Recognition, vol. 44, no. 2, pp. 375-385, Feb. 2011.

[4] B. Zhang, Y. Gao, S. Zhao, and J. Liu, "Local derivative pattern versus local binary pattern: Face recognition with high-order local pattern descriptor, "IEEE Trans. Image Process., vol. 19, no. 2, pp. 533-544, Feb. 2010.

[5] T. Jabid, M. H. Kabir, and O. Chae, "Local directional pattern (LDP)—A robust image descriptor for object recognition," in Proc.Seventh IEEE Int. Conf. Advanced Video Signal Based Surveillance, Boston, MA, Aug. 29-Sept. 1 2010, pp. 482-487

[6] Z. H. Guo, L. Zhang, and D. Zhang, "A completed modeling of local binary pattern operator for texture classification,” IEEE Trans. Image Process., vol. 19, no. 6, pp. 1657-1663, Jun. 2010.

[7] Vinh Dinh Nguyen, Duc Dung Nguyen, Thuy Tuong Nguyen, Vinh Quang Dinh and Jae Wook Jeon "Support Local Pattern and its Application to Disparity Improvement and Texture Classification" IEEE Trans.vol 24,No 2,feb 2014.

[8] J. Fierrez-Aguilar, N. Alonso-Hermira, G.Moreno-Marquez, and J. Ortega-Garcia, "An off-line signature verification system based on fusion of local and global information," in Proc. Workshop Biometric Authentication,Springer LNCS-3087, May 2004, pp. 298-306.

[9] N. VenkateswaraRao, Dr. A. Srikrishna, Dr. B. Raveendra Babu and G. Rama Mohan Babu "An Efficient Feature extraction and Classification of Handwritten digits using Neural Networks" in(IJCSEA) Vol.1, No.5, October 2011.

[10] Zhang, J., Marsza lek, M., Lazebnik, S., Schmid, C.: Local features and kernels for classification of texture and object categories: A comprehensive study. International Journal of Computer Vision $73(2), 213\{238(2007)$

[11] T. Randen, J. H. Husoy, Filtering for texture classification: A comparative study, IEEE Transactions on Pattern Analysis and Machine Intelligence 21 (1999) 291-310.

[12] L. Liu, B. Yang, P. Fieguth, Z. Yang, and Y. Wei, "BRINT: A Binary Rotation Invariant and Noise Tolerant Texture Classification," in Proc. IEEE Transactions on Image Processing vol. 23, NO. 7 , JULY 20143071 
International Journal of Artificial Intelligence and Applications (IJAIA), Vol. 7, No. 6, November 2016

\section{Authors}

Mr.Vickram pentyala is currently pursuing his bachelors degree B.Tech(CSE) from RVR \& JC College of engineering. His research interest includes algorithms, web security, computer vision and machine intelligence.

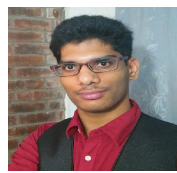

Dr.A. Srikrishna received the Ph.D degree from JNTUK,Kakinada in 2010, M.Tech degree in Computer Science from Jawaharlal Nehru Technological University (JNTU) in 2003, AMIE degree in Electronics \& Communication Engineering from Institution of Engineers; Kolkatta in 1990. She has 22 years of teaching experience, presently she is working as a Professor and Head, Dept of IT at RVR \& JC College of Engineering, Guntur. Her research interest includes Image Processing and Pattern Recognition. She has more than 30 Publications in various International Journals and Conferences. She is Associate member of IE (I) and member of CSI.

D. Swapna received M.Tech in CST from RVR \& JC College of Engineering, from ANU, in 2016 and B.Tech in IT from RVR \& JC College of Engineering, from ANU, in 2014 . Her research interest includes Image Processing and Pattern Recognition.

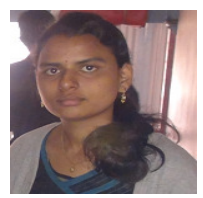

